\title{
Multipropósito de Hymenaea courbaril L. em uma área de manejo florestal comunitário na Amazônia
}

\author{
Daniele Lima da Costa ${ }^{1 *}$ Renato Bezerra da Silva Ribeiro ${ }^{2}$ Diego dos Santos Vieira ${ }^{3}$ Misael Freitas dos \\ Santos ${ }^{1}$ João Ricardo Vasconcellos Gama ${ }^{2}$ Bruno de Almeida Lima ${ }^{4}$ \\ ${ }^{1}$ Universidade Estadual do Centro Oeste, campus Irati, PR 153, Km 7 s/n, Riozinho - PR, 84500-000 \\ ${ }^{2}$ Universidade Federal do Oeste do Pará, Rua Vera Paz, s/n, Salé, Santarém-PA, 68040-255 \\ ${ }_{3}^{3}$ Universidade Federal dos Vales do Jequitinhonha e Mucuri, Rua Cruzeiro, Teófilo Otoni - MG, 39803-371 \\ ${ }^{4}$ Universidade do Estado de Santa Catarina, Centro de Ciências Agroveterinárias, Av. Luiz de Camões, 2090 - Conta Dinheiro, Lages - SC, 88520-000 \\ *Author for correspondence: danielelimadacosta@gmail.com \\ Received: March 2019 / Accepted: June 2019 / Published: June 2019
}

\section{Resumo}

$\mathrm{Na}$ Amazônia, numerosas espécies possuem valor comercial madeireiro e de produtos florestais não madeireiros (PFNM), havendo uma carência na quantificação deste segundo recurso e de meios que incentivem a utilização dos multiprodutos da floresta, de modo a aliar a produção dos mesmos. Com o intuito de investigar o potencial para multiprodutos de espécies florestais, este estudo analisou a estrutura populacional de Hymenaea courbaril L. em uma área de manejo comunitário e verificou o mercado de seus produtos não madeireiros na área urbana de Santarém-Pa. A área de estudo se localiza na Floresta Nacional do Tapajós no estado do Pará. A coleta de dados para análise da estrutura da floresta foi executada por meio de inventário $100 \%$, obtendo-se o diâmetro à altura do peito (DAP) $\geq 40 \mathrm{~cm}$, altura comercial e qualidade de fuste das árvores. A avaliação do mercado de PFNM foi realizada com a aplicação de entrevistas semiestruturadas com comerciantes das feiras de produtos naturais da cidade de Santarém-PA. A baixa densidade estimada é característica de populações de Hymenaea courbaril e sua distribuição espacial ocorreu agrupada na maior parte da área. A qualidade de fuste aliada à simulação de produtividade madeireira, assim como, a quantificação e valoração do produto seiva, são atraentes para um possível manejo. A estrutura da população de $H$. courbaril e a produção de madeira e seiva, demonstram potencial para comercialização. No entanto, o produto seiva tem pouca demanda de venda, e consequentemente, baixa rentabilidade financeira.

Palavras-chaves: Uso múltiplo, Produto florestal não madeireiro, comercialização, Florestas tropicais.

\footnotetext{
Abstract

In the Amazon, many species have commercial value and non-timber forest products (NTFPs); however, there is a lack of quantification of this second resource and of ways to encourage the use of multiproducts in the forest, in order to combine their production. In order to investigate the potential for multiproducts of forest species, this study analyzed the population structure of Hymenaea courbaril L. in an area of community forest management and verified the market of its non-timber products in the urban area of Santarém, Pará. The study area is located in the Tapajós National Forest in the state of Pará. Data collection for analysis of the forest structure was performed using a $100 \%$ forest inventory, obtaining the breast height diameter $(\mathrm{DBH}) \geq 40 \mathrm{~cm}$, commercial height and stem quality of the trees. The evaluation of the NWFP market was carried out with the application of a semi-
}

structured questionnaire with traders of the natural products fairs of the city of Santarém. The estimated low density is characteristic of populations of Hymenaea courbaril and its spatial distribution occurred grouped in most of the area. The quality of the wood combined with the simulation of wood productivity, as well as the quantification and valuation of the sap product, are attractive for a possible handling. The structure of the $H$. courbaril population and the production of wood and sap demonstrate potential for commercialization. However, the sap product has little demand for sale, and consequently, low financial profitability.

Keywords: Multiple use, Non-timber forest product, commercialization, Tropical forests.

\section{Introdução}

A espécie Hymenaea courbaril L. pertence à família Fabaceae e subfamília Caesalpinioideae (Lorenzi 2000). Na Amazônia, é conhecida como jatobá com árvores de médio a grande porte, ocorre principalmente em florestas primárias, em áreas de terra firme e várzea alta, estabelecendo-se com frequência em solos argilosos e pobres (Dendrogene 2004).

Dentre as espécies que ocorrem na Amazônia, $H$. courbaril é uma das que ganham destaque por atingir maiores alturas, até $65 \mathrm{~m}$ em florestas tropicais, e diâmetros (Silva 2010). Possui árvores de troncos retos e cilíndricos, de súber liso e de coloração cinza (Cipriano 2014). A floração e a frutificação têm início entre oito e doze anos de idade da planta e não são necessariamente anuais, os frutos amadurecem entre os meses de agosto e setembro (Costa et al. 2011).

A espécie é bastante utilizada por populações tradicionais devido suas diversas utilidades, e com mercado incentivado pelos vários produtos obtidos a partir desta (Lima 2009). Podendo ser aproveitadas todas as partes da árvore, como: sementes, frutos, resina, casca, seiva e folhas; que podem ser empregadas de diferentes formas, sendo usado tanto como fitoterápico quanto na culinária (Shanley e Medina 2005). O valor no uso de Produtos Florestais não Madeireiros é na maioria das vezes de importância considerável para as comunidades da floresta, pois além de proporcionar fontes de alimentos, medicamentos e renda, tem valor cultural ou espiritual (Colfer, 2008). Além disso, a utilização destes recursos como geradores de renda, poderá conciliar desenvolvimento econômico com conservação do ecossistema florestal e manutenção das populações em suas regiões de origem (Almeida et al. 2009).

Outro produto da espécie é a madeira, que possui alto valor comercial com grande aceitação no mercado local e 
externo, podendo ser empregada em obras hidráulicas, carroçarias, postes, tonéis, dormentes, construções variadas, móveis, laminados, tacos, entre outros usos (Costa et al. 2011). Espécies florestais com alto potencial para multiprodutos e diferentes formas de comercialização merecem atenção, informações obtidas em levantamentos sobre estrutura populacional de uma espécie servem de base para a realização de um manejo adequado e para sua proteção e recuperação (Freitas e Magalhães 2012); pois indicam como estão arranjadas em seu hábitat natural e fornecem informações sobre sua dinâmica sucessional (Callegaro et al. 2012).

Desse modo, com o intuito de verificar o comportamento populacional desta espécie em uma área de manejo comunitário, e assim, conhecer as expectativas de possíveis rendas pelos seus multiprodutos, este estudo objetivou analisar a estrutura populacional de Hymenaea courbaril L. e sua capacidade produtiva madeireira na Floresta Nacional do Tapajós, verificar o mercado de seus produtos não madeireiros em Santarém-PA e valorar os principais produtos da espécie comercializados na região.

\section{Materiais e métodos \\ Área de estudo}

O estudo foi realizado na Unidade de Produção Anual (UPA) $\mathrm{n}^{\circ} 10$, com 1.551,88 ha, localizada na Área de Manejo Florestal (AMF) Anambé, nos domínios da Floresta Nacional do Tapajós (FNT), município de Belterra, estado do Pará (Figura 1). O clima da região é do tipo Ami, com temperatura e precipitação média anual de $25,5^{\circ} \mathrm{C}$ e $1820 \mathrm{~mm}$, respectivamente (ALVARES et al. 2013). O solo predominante na região é do tipo Latossolo Amarelo Distrófico, profundos e bem drenados (Raimundo Junior et al. 2015; Bispo et al. 2016). A vegetação é classificada como Floresta Ombrófila Densa, caracterizando-se pela presença de árvores de grande porte e pela abundância de lianas lenhosas, palmeiras e epífitas (IBGE 2012).

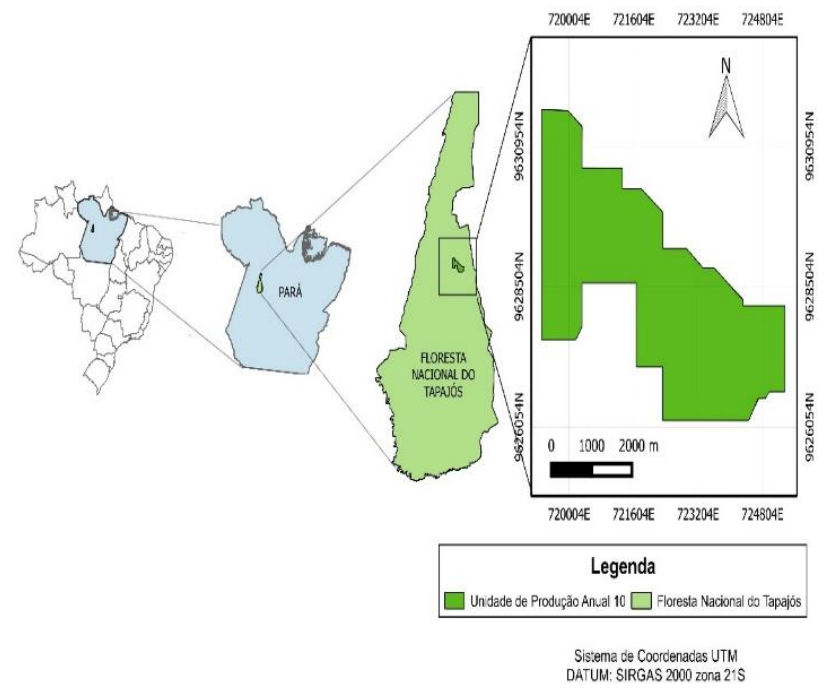

Figura 1. Mapa de localização da Unidade de Produção Anual (UPA) $n^{\circ} 10$, na área de manejo florestal comunitário na Floresta Nacional do Tapajós-PA, Amazônia.

\section{Coleta de dados}

Os dados foram coletados a partir de um inventário $100 \%$, com mapeamento, em coordenadas cartesianas (X, $\mathrm{Y})$, de todas as árvores de Hymenaea courbaril L. com diâmetro a 1,30 m do solo (DAP) igual ou superior a 40 $\mathrm{cm}$. Toda a área da UPA $\mathrm{n}^{\circ} 10$ foi dividida em 16 Unidades de Trabalho (UTs), com área média de 96,9 hectares. Afim de facilitar a numeração e o mapeamento dos representantes da espécie em cada UT, foram abertas picadas paralelas na direção norte-sul, equidistantes $50 \mathrm{~m}$. Em cada picada foram colocadas balizas a cada $25 \mathrm{~m}$, com a respectiva metragem em relação à origem, para posterior registro das coordenadas $\mathrm{Y}$ de cada árvore (VIEIRA et al. 2017).

A coordenada $\mathrm{X}$ de cada indivíduo foi obtida por meio do número da picada e da distância de cada árvore em relação a ela. Ao final de cada picada, movia-se para a picada seguinte e executava-se a mesma rotina de trabalho, e assim sucessivamente, até completar a última picada das UTs (VIEIRA et al. 2017). As variáveis dendrométricas coletadas foram: circunferência à $1,30 \mathrm{~m}$ do solo (CAP), altura comercial $\left(\mathrm{H}_{\mathrm{c}}\right)$ e qualidade de fuste $\left(\mathrm{Q}_{\mathrm{F}}\right)$, que apresentou três classes, a saber: $\mathrm{QF}_{1}=$ árvore com fuste de boa qualidade e aproveitamento de 80 a $100 \% ; \mathrm{QF}_{2}=$ árvores com pequenas tortuosidades ao longo o fuste $\mathrm{e}$ aproveitamento de 50 a 79\%; $\mathrm{QF}_{3}=$ árvores com tortuosidades acentuadas e aproveitamento inferior a $50 \%$ (Amaral et al. 1998).

\section{Estrutura populacional e distribuição espacial}

Os parâmetros fitossociológicos da estrutura horizontal foram calculados conforme Souza e Soares (2013). A estrutura interna foi avaliada de acordo com Jardim e Hosokawa (1986). A estrutura diamétrica foi obtida por meio do cômputo das árvores em classes diamétricas com amplitude de $10 \mathrm{~cm}$, a partir do diâmetro mínimo de inclusão das árvores (Soares et al. 2011). O padrão espacial das árvores foi obtido por meio da função $\mathrm{K}$ (s) de Ripley. O cálculo de K(s) foi realizado em função de um círculo com raio $s$ de $5 \mathrm{~m}$ centrado em cada árvore, em que o número de eventos presentes na área desse círculo foi contado. Variando o raio a uma distância máxima de $3.000 \mathrm{~m}$, detectou-se o padrão espacial da espécie em diferentes escalas de distância, desta forma a função $\mathrm{K}(\mathrm{s})$ de Ripley avaliou a relação existente entre pares de eventos a cada $5 \mathrm{~m}$ até a distância máxima de $3.000 \mathrm{~m}$, considerada a metade do maior eixo longitudinal da área (Vieira et al. 2017).

Casos particulares ocorreram quando as árvores estavam próximas às bordas da área, pois como K(s) é acumulativa e computa todas as distâncias entre todos os eventos, árvores próximas à borda de raio maior que o limite do mapa não poderiam ser interpretadas como se não houvesse vizinhos (Capretz et al. 2012). As árvores existiam, mas por estarem fora dos limites da área de estudo não foram computadas. Por conseguinte, o número de árvores vizinhas a eventos próximos aos limites do mapa seria mais baixo do que para as demais, ocasionando um viés no cálculo do estimador de $\mathrm{K}(s)$. À vista disso, utilizou-se a o estimador da função $\widehat{\mathrm{K}}(\mathrm{s})$ com correção isotrópica de bordadura proposta por Ripley (1979).

$$
\mathrm{K}(\mathrm{s})=\frac{1}{\hat{\lambda} g} \sum_{\mathrm{i}=1}^{\mathrm{g}_{\mathrm{i}}} \sum_{\mathrm{i}=1}^{\mathrm{g}_{\mathrm{i}}} \mathrm{W}_{\mathrm{I}}^{-1}\left(\left\|\mathrm{X}_{\mathrm{i}}-\mathrm{X}_{\mathrm{j}}\right\| \leq \mathrm{s}\right) \text {, para } \mathrm{i} \neq \mathrm{j} \text { e } \mathrm{s}>0
$$

em que: $\mathrm{g}$ é o número de árvores na região de estudo; $\mathrm{X}_{\mathrm{i}} \mathrm{e}$ $\mathrm{X}_{\mathrm{j}}=$ coordenadas dos pontos do mapa; $\left\|\mathrm{X}_{\mathrm{i}}-\mathrm{X}_{\mathrm{j}}\right\|=$ distância euclidiana entre a localização $X_{i}$ e $X_{j} ; s=$ vetor arbitrário de distância; $W_{I} I\left(X_{i}, X_{j}\right)=$ função de correção para efeito de borda, que representa a proporção da circunferência com centro em $\mathrm{X}_{\mathrm{i}}$ e com raio $\left\|\mathrm{X}_{\mathrm{i}}-\mathrm{X}_{\mathrm{j}}\right\|$ que está fora da região de estudo; $\hat{\lambda}=\mathrm{n} /|\mathrm{A}|=$ número de árvores dividido pela área da região de estudos, sendo um estimador não viciado da intensidade do processo; e $\mathrm{I}(\mathrm{U})$ = função 
indicadora que assume valor 1 sempre que a condição $U$ for verdadeira, e zero quando é falsa.

Em seguida, para analisar os dados graficamente, foi construído um envelope de confiança por meio de 500 simulações Monte Carlo, realizas por meio do modelo de completa aleatoriedade espacial (CAE). A função K(s) foi calculada para os resultados das simulações, armazenando-se os valores mínimos e máximos da estimativa de $\mathrm{K}(\mathrm{s})$, utilizados para gerar intervalos de confiança a 99\% de probabilidade (CAPRETZ et al. 2012). Todavia, para melhor visualização das características de CAE, os valores da função $\mathrm{K}(\mathrm{s})$ foram transformados para L(s), de acordo com a expressão seguir, e distribuídos graficamente, em que os eixos das abscissas e ordenadas representam, respectivamente, as distâncias $s$ acumuladas e os valores transformados da função K(s) (RIPLEY, 1979).

$$
L(s)=\sqrt{\frac{K(s)}{\pi}}
$$

Se os valores de $\mathrm{L}(\mathrm{s})$ permanecem dentro dos envelopes de confiança identificados por duas linhas limites pontilhadas, uma positiva e outra negativa, aceitase a hipótese CAE e o padrão espacial é completamente aleatório, caso contrário rejeita-se a hipótese e assume-se que o padrão espacial dos indivíduos é agregado, quando passar do limite superior do envelope, e regular, quando passar do limite inferior (Ripley, 1979; Machado et al. 2012).

\section{Volumetria e estoque de colheita}

A volumetria da espécie foi avaliada através da distribuição do volume nas classes diamétricas. $\mathrm{O}$ volume comercial com casca $\left(\mathrm{v}_{\mathrm{c} / \mathrm{c}}\right)$ foi calculado a partir da equação abaixo, ajustada para a UPA n ${ }^{\circ} 10$ (Coomflona 2015).

$$
\begin{aligned}
& \mathrm{v}_{\mathrm{c} / \mathrm{c}}=((-8,9525703778108)+1,88437411839699 \\
& \text { e * } \left.\operatorname{Ln}(\text { DAP })+0,807002832127167 * \operatorname{Ln}\left(h_{c}\right)\right)
\end{aligned}
$$

em que: $\mathrm{v}_{\mathrm{c} / \mathrm{c}}=$ volume com casca, em $\mathrm{m}^{3}$; DAP = diâmetro medida à $1,30 \mathrm{~m}$ do solo, em $\mathrm{cm} ; \mathrm{h}_{\mathrm{C}}=$ altura comercial, em metros; $\mathrm{Ln}=$ logaritmo neperiano.

A estimativa do estoque de colheita de madeira da UPA $n^{\circ} 10$ foi realizada considerando-se todas as árvores com DAP $\geq 50 \mathrm{~cm}$. A seleção das árvores para corte e remanescente considerou as recomendações dos órgãos ambientais federais e estaduais, a saber: Instrução Normativa $\mathrm{n}^{\circ} 05$ de 11/12/2016 do Ministério do Meio Ambiente, Instrução Normativa ${ }^{\circ} 07$ de 27/09/2006 da Secretaria do Estado de Ciência, Tecnologia e Meio Ambiente do estado do Pará e Norma de Execução no 01 de 24/04/2007 do Instituto Brasileiro de Meio Ambiente e dos Recursos Naturais Renováveis. Logo, foram selecionadas as árvores com DAP $\geq 50 \mathrm{~cm}$ que apresentaram $\mathrm{QF}_{1}$ e $\mathrm{QF}_{2}$, foram mantidos $10 \%$ do número de árvores selecionadas para a colheita por espécie, respeitou-se a ocorrência mínima de 3 árvores por espécie por 100 ha e a intensidade máxima de corte (IMC) de 30 $\mathrm{m}^{2} \mathrm{ha}^{-1}$.

\section{Comercialização de produto florestal não madeireiro}

A identificação dos produtos florestais não madeireiros (PFNM) de Hymenaea courbaril L. comercializados na região foi realizada por meio de entrevista semiestruturadas realizadas em três feiras públicas do município de Santarém, estado do Pará, a saber: Mercadão 2000, Candilha e Cohab. A entrevista procurou responder as seguintes perguntas: quais os PFNM mais procurados; qual o motivo da procura; e quais os impedimentos encontrados pelos comerciantes com relação à venda dos PFNM.

\section{Valoração dos produtos}

A valoração e o potencial de produção da seiva de Hymenaea courbaril L. foi realizada considerando-se uma produtividade de 13,2 litros arv. $^{-1}$ (LIMA 2007) e o valor de $\mathrm{R} \$ 15,00$ litro $^{-1}$, de acordo com os comerciantes entrevistados, para todas as árvores possíveis com DAP $\geq$ $30 \mathrm{~cm}$ em uma UT de 100 hectares. A valoração da madeira, por sua vez, foi obtida considerando-se todas as possíveis árvores com DAP $\geq 70 \mathrm{~cm}$, preço de $\mathrm{R} \$ 395,00$ $\mathrm{m}^{-3}$ e, também, uma UT de 100 hectares. As equações abaixo foram empregadas para o cálculo do valor monetário dos PFNM e de fustes de Hymenaea courbaril L, respectivamente (GAMA, 2004; PINHEIRO et al. 2019).

$$
\begin{gathered}
\mathrm{VM}_{\mathrm{PFNM}}=\sum_{\mathrm{i}=1}^{\mathrm{S}} \mathrm{PM}_{\mathrm{i}} \cdot \mathrm{NA}_{\mathrm{i}} \cdot \mathrm{PC}_{\mathrm{i}} \\
\mathrm{VM}_{\mathrm{FEC}}=\sum_{\mathrm{i}=1}^{\mathrm{S}} \mathrm{VFE}_{\mathrm{i}} \cdot \mathrm{P}_{\mathrm{i}} .
\end{gathered}
$$

em que: VMPFNM = valor monetário dos PFNM da espécie comercializada, em $\mathrm{R} \$ .100 \mathrm{ha}^{-1} ; \mathrm{PM}_{\mathrm{i}}=$ produção média anual do $i$-ésimo PFNM, em litros; $\mathrm{NA}_{\mathrm{i}}=$ número de árvores produtivas $(\mathrm{DAP} \geq 30 \mathrm{~cm})$, em árv. $100 \mathrm{ha}^{-1} ; \mathrm{PC}_{\mathrm{i}}=$ preço de comercialização na floresta do $i$-ésimo PFNM, em $\mathrm{R} \$ \mathrm{~L}^{-1} ; \mathrm{VM}_{\mathrm{FEC}}=$ valor monetário de fuste das espécies, em $\mathrm{R} \$ .100 \mathrm{ha}^{-1} ; \mathrm{VFE}_{\mathrm{i}}=$ volume de fuste do estoque de colheita $(\mathrm{DAP} \geq 70 \mathrm{~cm})$ da $i$-ésima árvore selecionadas para corte, em m${ }^{3} .100 \mathrm{ha}^{-1} ; \mathrm{P}_{\mathrm{i}}=$ preço da madeira da $i$ ésima árvore em pé, em $\mathrm{R} \$ . \mathrm{m}^{-3}$; e $\mathrm{S}=$ número de espécies.

Os cálculos estruturais e de valoração foram realizados com o auxílio de planilhas eletrônicas no Microsoft Excel 2016, enquanto que a função K de Ripley e gráficos foram calculados e obtidos por meio do software $\mathrm{R}$ versão 3.3.2, utilizando-se os pacotes splancs (ROWLINGSON e DIGGLE, 2016)

\section{Resultados}

\section{Estrutura populacional e distribuição espacial}

Foram registradas 437 árvores de $H$. courbaril, o que correspondeu a uma densidade e dominância de 0,28 arv. $\mathrm{ha}^{-1}$ e $0,19 \mathrm{~m}^{2} \mathrm{ha}^{-1}$, respectivamente. A população apresentou uma distribuição diamétrica com tendência à normalidade (Figura 1), com maior concentração de árvores nas classes diamétricas centrais, não apresentando, dessa forma, a ocorrência da típica curva exponencial negativa (J-invertido) característica de florestas inequiâneas. 


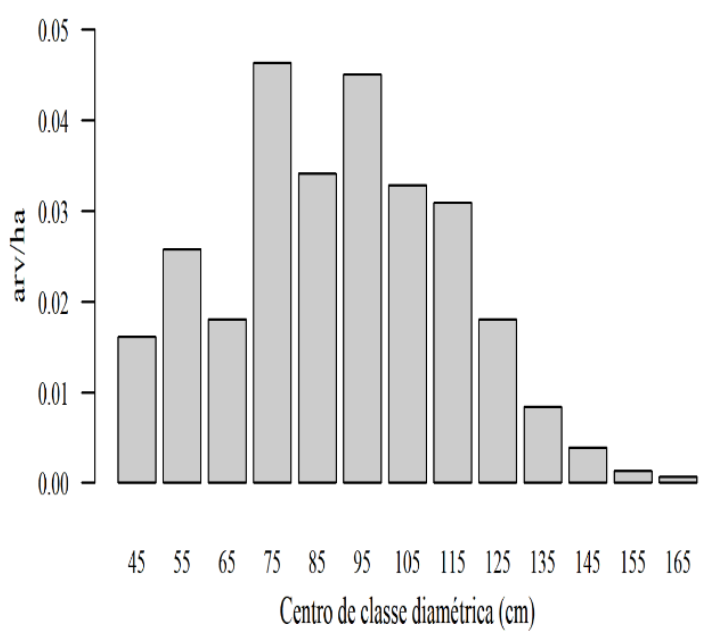

Figura 1. Distribuição diamétrica da espécie Hymenaea courbaril L. em uma área de manejo florestal comunitário na Floresta Nacional do Tapajós no estado do Pará, Amazônia.

Pela análise da função $\mathrm{K}$ de Ripley, foi possível observar que a espécie não apresentou um único comportamento na sua distribuição espacial, onde variou em função da distância considerada. Na escala até $150 \mathrm{~m}$, aproximadamente, a espécie apresenta padrão espacial aleatório; desse ponto até $1800 \mathrm{~m}$, aproximadamente, a espécie se comporta de modo agrupado e a partir dessa escala apresenta distribuição uniforme (Figura 2). Com isto, a hipótese de CAE foi rejeitada na maior área de ocorrência da espécie, predominando a forma agrupada.

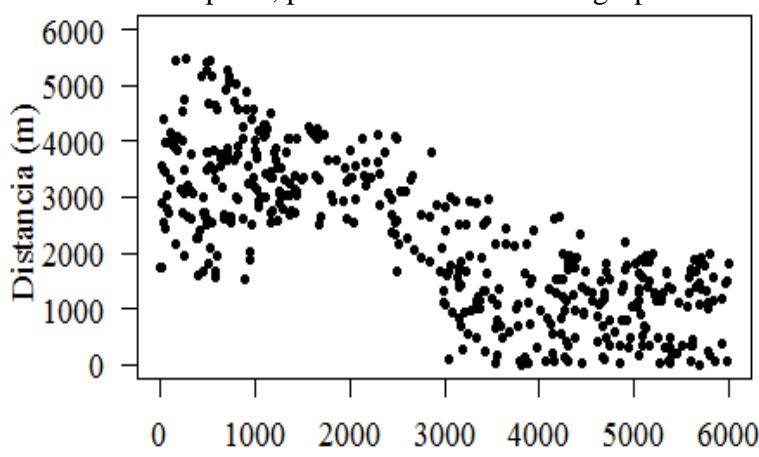

Distancia (m)

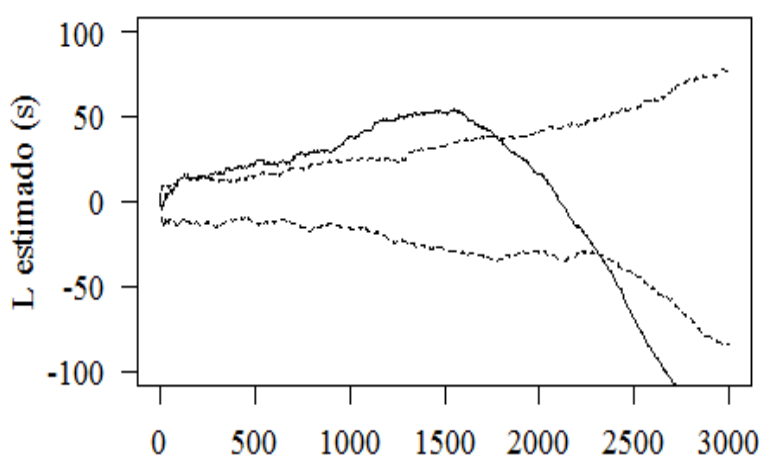

Distancia (m)

Figura 2. Padrão de distribuição espacial observado por meio da função K de Ripley para a espécie Hymenaea courbaril L. em uma área de manejo florestal comunitário na Floresta Nacional do Tapajós no estado do Pará, Amazônia.

\section{Volumetria e estoque de colheita}

$\mathrm{O}$ volume correspondente para as árvores da espécie com DAP $\geq 40 \mathrm{~cm}$ foi estimado em $2,58 \mathrm{~m}^{3} \mathrm{ha}^{-1}$. Para árvores que compõe o estoque de colheita, $\mathrm{DAP} \geq 50 \mathrm{~cm}$, o volume obtido foi de $2,54 \mathrm{~m}^{3} \mathrm{ha}^{-1}$. Ao considerar as árvores com qualidade de fuste 1 e $2(\mathrm{DAP} \geq 50 \mathrm{~cm})$, que na maioria dos planos de manejo são requeridas para seleção da colheita, o volume estimado foi de $2,38 \mathrm{~m}^{3} \mathrm{ha}^{-}$ 1 .

A distribuição volumétrica da espécie demonstrou padrão de distribuição comumente encontrado para população de floresta ou de uma espécie, evidenciando maior volume nas classes centrais, sendo as classes dos extremos com menores volumes (Figura 3), devido ao fato de as classes centrais apresentarem seus diâmetros não tão elevado quanto as últimas classes $(\geq 135 \mathrm{~cm})$, mas que detêm as maiores quantidades de árvores, proporcionando assim, maior concentração volumétrica.

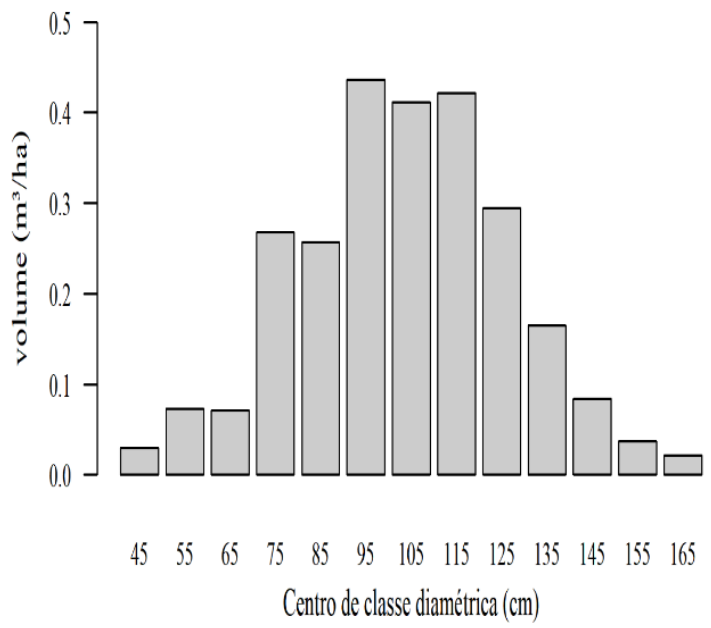

Figura 3. Distribuição volumétrica da espécie Hymenaea courbaril em uma área de manejo florestal comunitário na Floresta Nacional do Tapajós no estado do Pará, Amazônia.

As árvores de $H$. courbaril apresentaram em sua maioria fustes que podem ter boa aceitação no mercado madeireiro da região, ou seja, qualidade de fuste 1 e 2 (figura 4A), totalizando 94,27\% do número de árvores. Ao observar a distribuição dos diâmetros em cada classe de fuste (Figura 4B), notou-se que as árvores com qualidade de fuste 2 tem mais variação diamétrica, abrangendo os menores e maiores diâmetros. A qualidade de fuste 1 tem tendência semelhante, no entanto, menos acentuada. Já a qualidade de fuste 3 , concentra árvores com DAP $\geq 55 \mathrm{~cm}$ e com menor variação. Estes resultados demonstram ainda, que tanto as árvores com DAP $\geq 50 \mathrm{~cm}$, como com DAP $<$ $50 \mathrm{~cm}$, apresentam fuste retilíneo ou com pouca tortuosidade, em sua maioria. 

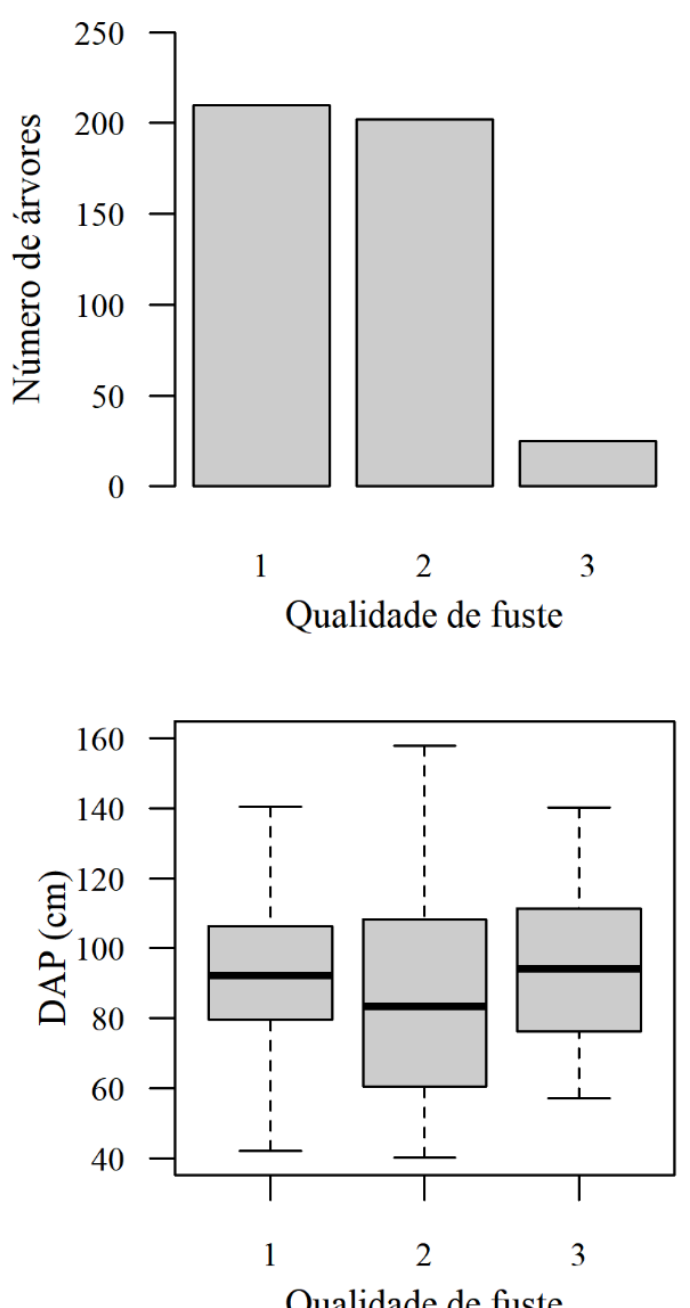

Figura 4. Quantificação das árvores de Hymenaea courbaril L. quanto a sua classificação de qualidade de fuste e distribuição dos diâmetros por qualidade de fuste, em uma área de manejo florestal comunitário na Floresta Nacional do Tapajós no estado do Pará, Amazônia.

A avaliação do estoque de colheita de madeira evidenciou 0,25 arv. ha-1 com volume médio de $0,0062 \mathrm{~m}^{3}$ ha $^{-1}$. A simulação de uma colheita de madeira na UPA $\mathrm{n}^{\circ} 10$, considerando-se a legislação em vigência, resultou como remanescentes $17,40 \%$ das árvores do estoque de colheita. Assim, considerando uma UT de 100 ha, foi estimado 24,94 arv. $100 \mathrm{ha}^{-1}$ e 238,58 $\mathrm{m}^{3} 100 \mathrm{ha}^{-1}$ passíveis de serem colhidos.

\section{Estimativa e valoração dos produtos madeireiros e não madeireiros}

A estimativa da produção madeireira da espécie foi realizada para as árvores passíveis de colheita, considerando o volume de 238,5805 $\mathrm{m}^{3} 100 \mathrm{ha}^{-1}$. Como o preço da madeira em tora comercializado pela COOMFLONA é de $\mathrm{R} \$ 395,00 \mathrm{~m}^{-3}$, foi obtido o valor de 294.239,00 R $\$ 100 \mathrm{ha}^{-1}$. O potencial de produção da seiva de $H$. courbaril foi estimado em 373,12 litros $100 \mathrm{ha}^{-1}$, o preço médio na área urbana de Santarém relatada pelos entrevistados foi de $\mathrm{R} \$ 15,00$ litro $^{-1}$, totalizando um valor monetário estimado para comercialização de 5.596,80 R\$ $100 \mathrm{ha}^{-1}$.
No entanto, é importante considerar que estas são estimativas que não levam em consideração os custos de operação, e que foram realizadas a partir $\mathrm{B} n$ processo de amostragem em uma floresta de alta vaı rabilidade, a qual não é homogênea em termos de estoque de produção, e ainda, características peculiares de determinadas regiões podem elevar custos de colheita. Contudo, as estimativas de produção e valoração, poderá dar embasamento para tomada de decisão em manejar determinados produtos.

\section{Comercialização do produto florestal não madeireiro no mercado de Santarém, $P A$}

Os principais PFNM obtidos da espécie $H$. courbaril comercializados nas feiras de Santarém-PA são a seiva e a casca, sendo a seiva relatada como o produto mais comercializado em todos os estabelecimentos. Segundo os comerciantes, as pessoas têm preferência por esse produto devido sua praticidade, já que pode ser ingerida sem outros preparos. Algumas vezes eles fazem a indicação do produto quando solicitado, mas relataram que na maioria dos casos as pessoas que o procuram já conhecem suas propriedades.

Todos os estabelecimentos entrevistados comercializam a seiva, sendo que $80 \%$ dos entrevistados relataram que há pouca procura pelo mesmo. Logo, os entrevistados destacaram que a venda do produto é pouco rentável devido à baixa frequência de procura do mesmo. A maioria dos compradores são locais, mas os comerciantes também recebem turistas de outros estados e do exterior.

\section{Discussão}

A densidade de 0,28 arv. ha ${ }^{-1}$ registrada para a espécies H. courbaril foi considerada mediana. Outros estudos realizados na Amazônia registraram densidades que variaram de 0,07 a 0,59 arv. ha ${ }^{-1}$ (Pinheiro et al. 2007; Lima et al. 2009; Silva et al. 2009; Schwartz et al. 2014; Sontag, 2017), corroborando não só os resultados desse estudo mais as afirmativas de Silva et al. (2009) e Shanley e Medina (2005), que apontaram que, geralmente, a espécie possui menos de 1,0 arv. ha ${ }^{-1}$. A variação de densidade pode ser atribuída às diferentes intensidade amostrais e níveis de inclusão dos inventários, além de condições edáficas da área de estudo (Wadt, 2005). A distribuição diamétrica tendendo à normalidade demonstrou que, apesar do diâmetro mínimo de inclusão do inventário (DAP $\geq 40 \mathrm{~cm}$ ), a população possui ou possuiu problemas de regeneração, pois as menores classes diamétricas, ou seja, aquelas com centro de classe de 45 , 55 e $65 \mathrm{~cm}$, possuem baixa densidade. Todavia, não é possível afirmar que a regeneração vem recuperando-se ao longo do tempo, pois o inventário não abrangeu as classes menores, isto é, aquelas com DAP $<40 \mathrm{~cm}$.

A distribuição tendendo à normalidade é consequência do baixo recrutamento de plantas jovens, ocasionado, possivelmente, pela baixa produção de sementes e taxa de germinação, além da mudança do estágio sucessional da floresta de inicial para mais avançado. Ainda podem ser resultados do ritmo lento de desenvolvimento das árvores, o que é associado à espécies de ciclo longo com pequeno número de sementes e germinação lenta, e das alterações climáticas, as quais tem gerado condições de menor precipitação, o que afeta a capacidade de alguma espécies de reproduzirem-se e estabelecerem árvores jovens (VIEIRA et al. 2018). Todos esses eventos, provavelmente ocorreram décadas atrás na UPA n ${ }^{\circ} 10$, fazendo com que a 
estrutura diamétrica a partir de $40 \mathrm{~cm}$ apresente esse comportamento.

Ademais, do ponto de vista madeireiro, a maior densidade nos centros de classe de 75 a $135 \mathrm{~cm}$ é atraente, pois há maior disponibilidade de madeira. Todavia, a baixa disponibilidade de árvores nas classes abaixo de $50 \mathrm{~cm}$, sugerem que uma colheita intensa pode afetar negativamente a densidade, por meio de impactos decorrentes das atividades de derruba, e, consequentemente, o estoque de crescimento da espécie, influenciando na capacidade de recuperação da mesma para ciclos de corte de 30 anos. O padrão espacial dependente da distância, isto é, ora agregado, ora aleatório e ora regular é característico de espécies tropicais. As variações de padrão na escala de distância são geralmente relacionadas ao porte arbóreo das árvores, pois árvores mais jovens tendem a ocorrer mais próximas umas das outras, formando agregados. À medida que elas crescem ocorre aumento da competição e poucas alcançam os maiores diâmetros, ficando mais distantes umas das outras, o que leva a distribuição aleatória (Araújo et al. 2014)

Ebert et al. (2016) encontraram padrões semelhantes para uma população de $M$. itauba no sul da Amazônia e atribuíram esse comportamento ao crescimento vegetativo, dispersão zoocórica e disponibilidade de recursos, como nutrientes leves e essenciais, que resulta em maiores níveis de competição. Ainda segundo Shanley e Medina (2005), Seidler e Plotkin (2006) e Negrini et al. (2012), a síndrome de dispersão de uma espécie contribui fortemente para sua distribuição espacial e, no caso de $H$. courbaril, que apresenta dispersão zoocórica, o padrão agregado é fortemente influenciado por sua síndrome de dispersão.

A maior ocorrência de árvores com fuste do tipo 1e 2, aliado ao estoque de colheita simulado para o manejo madeireiro da espécie e a análise de sua distribuição diamétrica, demonstram o potencial da área para um possível manejo madeireiro. De acordo com Silva (2010), a forma do fuste sem achatamento e curvatura são determinantes para se obter boa qualidade e rendimento satisfatório no processo de madeira serrada. Com isto, as toras da espécie obtidas com uma possível colheita teriam boa aceitação no mercado por serem, em sua maioria, de forma retilínea ou com pouca tortuosidade.

Outro fator importante, em relação ao manejo desta espécie é a ocorrência de sua distribuição espacial do tipo agrupada na maior parte da área, que pode ser um fator que contribua para o aproveitamento de seus recursos, pois essa distribuição pode facilitar as operações de manejo, uma vez que as árvores tendem a estarem mais próximas umas da outras, contribuindo assim, para um menor deslocamento, diminuição de custos e tempo das atividades. É importante frisar que o conhecimento da estrutura populacional de determinada espécie de interesse de grande atratividade ao mercado é importante para a tomada de decisão do seu manejo, pois permite um planejamento adequado que possa garantir sua permanência na floresta.

A baixa procura e consequentemente pouca rentabilidade nas vendas dos produtos não madeireiros, casca e seiva, pode ser um fato atrelado a falta de conhecimento por grande parte da população urbana sobre as utilidades medicinais e por não terem o hábito de fazer uso dos produtos da floresta, que se apresentam de forma rústica e sem industrialização, vendidos em algumas feiras de produtos naturais da cidade. A falta de incentivo para divulgação dos produtos da floresta contribuem para este cenário e como bem colocado por Pedrozo et al. (2011), o fortalecimento de uma atividade econômica de produtos florestais não madeireiros enfrenta entraves, tornando-se incipiente e gerando poucas oportunidades financeiramente viáveis.

Observa-se que o valor obtido com a valoração madeireira é bastante expressivo em relação à valoração do produto seiva, o que pode muitas das vezes ser um fator para que não se leve em conta o manejo de produtos florestais não madeireiros, realizado em muitos casos apenas manejo madeireiro. No entanto, a análise da renda dos possíveis produtos que podem ser obtidos de uma espécie é essencial para demonstrar outros aproveitamentos que não só a madeira, e assim, alia-los.

\section{Conclusão}

A estrutura da população de Hymenaea courbaril L. e a quantificação e valoração de seus produtos madeira e seiva, demonstraram potencial para comercialização destes recursos, podendo viabilizar o seu manejo. Contudo, há pouca demanda pelo produto seiva no mercado de Santarém-PA, e consequentemente, considerando este cenário, este produto não é atrativo para os comerciantes.

\section{Referências}

Almeida NA, Bittencourt AM, Santos AJ, Eisfeld CL.E, Souza VS (2009) Evolução da produção e preço dos principais produtos florestais não madeireiros extrativos do Brasil. Revista Cerne, 15(3): 282-287.

Alvares CA, Stape, JL, Sentelhas PC, Gonçalves JLM, Sparovek G (2013) Koppen's climate classification map for Brazil. Meteorologische Zeitschrift, 22 (6): 711-728.

Amaral, P. et al. (1998) Florestas para sempre: um manual para a produção de madeira na

Araújo E, David H, Péllico S, Morais V, Scolforo J (2014) Padrão espacial de espécies arbóreas em fragmento de floresta estacional semidecidual. Revista de Ciências Agrarias 57:166-171.

Bispo PC, Santos JR, Valeriano MM, Graça PMLA, Balzter H, França H, Bispo PC (2016) Predictive Models of Primary Tropical Forest Structure from Geomorphometric Variables Based on SRTM in the Tapajós Region, Brazilian Amazon. Plos One, 11(4): 1-13.

Callegaro RM, Longhi SJ, Araujo ACB, Kanieski MR, Floss PF, Gracioli CR (2012) Estrutura do componente arbóreo de uma floresta estacional decidual ripária em Jaguari, RS. Revista Ciência Rural, 42(2): 305-311.

Capretz, RL; Batista, JLF; Sotomayor, JFM; Cunha, CR; Nicoletti, MF; Rodrigues, RR (2012) Padrão espacial de quatro formações florestais do estado de São Paulo, através da função K de Ripley. Ciência Florestal, 22(3): 551-565.

Cipriano J, Martins L, Deus MDSM, Peron APO (2014) Gênero Hymenaea e suas espécies mais importantes do ponto de vista econômico e medicinal para o Brasil. Caderno de Pesquisa, 26(2): 41-51.

Colfer CJP (2008) Human Health and Forests: A Global Interdisciplinary Overview of Issues, Practice and Policy, Earthscan/CIFOR, London. 
Costa WS, Souza AL, Souza PB (2011) Ecologia, Manejo, Silvicultura e Tecnologia de Espécies Nativas da Mata Atlântica. Engenharia Florestal- DEFUFV. Espécies Nativas da Mata Atlântica. 21p.

Dendrogene (2004) Jatobá, / Hymenaea courbaril /. Belém, PA: Embrapa Amazônia Ocidental: Projeto Dendrogene.

Ebert A, Costa RB, Brondani G (2016). Spatial distribution pattern of Mezilaurus itauba (Meins.) Taub. Ex Mez. in a seasonal forest area of the southern Amazon, Brazil. iForest - Biogeosciences and Forestry, 9:497-502.

Freitas WK, Magalhães LMS (2012) Métodos e Parâmetros para Estudo da Vegetação com Ênfase no Estrato Arbóreo. Floresta e Ambiente, 19(4): 520-540.

Gama JRV (2004) Proposta metodológica para avaliação monetária de produto florestal madeireiro e não madeireiro. Carajás: IAVRD/Salobo Metais. 13p.

Instituto Brasileiro de Geografia e Estatística - IBGE (2012) Manual técnico da vegetação brasileira. Rio de Janeiro: $275 \mathrm{p}$.

Jardim FCS, Hosokawa RT (1986) Estrutura da floresta equatorial úmida da Estação Experimental de Silvicultura Tropical do INPA. Acta Amazônica, 16: 411-508.

Lima A, Azevedo KS, Campos CA, Tavares US, Rocha AA (2007) Manejo da seiva do jatobá (Hymenaea Courbaril L.) por famílias tradicionais na Reserva Extrativista Chico Mendes, Acre-Brasil. In: VIII Congresso de Ecologia do Brasil, Caxambu, Minas Gerais.

LIMA A.F (2009) Estrutura populacional do jatobá (Hymenaea courbaril 1.) na reserva extrativista Chico Mendes (resex Chico Mendes), município de brasileia/Acre, sudoeste da Amazônia. In: Congresso de ecologia do brasil, São Lourenço, Minas Gerais.

Lorenzi H (2000) Árvores brasileiras: manual de identificação e cultivo de plantas arbóreas nativas do Brasil. 2.ed. Nova Odessa: Plantarum. 368p.

Machado SA, Santos AAP, Zamin N, Nascimento RGM (2012) Distribuição espacial de um fragmento de Floresta Ombrófila Mista Montana, Ciência Rural.

Negrini M, Aguiar MD, Vieira CT, Silva AC, Higuchi P (2012) Dispersão, distribuição espacial e estratificação vertical da comunidade arbórea em um fragmento florestal no planalto catarinense. Revista Árvore 36(5): 919-929.

Pedrozo EA, Silva TN, Sato SAS, Oliveira NA (2011) Produtos Florestais Não Madeiráveis (PFNMS): as Filières do Açaí e da Castanha da Amazônia, Revista de Administração e Negócios da Amazônia, 3(2): 88-112.

Pinheiro JC, Gama JRV, Oliveira FA, Ribeiro, RBS (2019) Fitossociologia e expectativa de renda com produtos florestais madeireiros e não madeireiros em assentamento no Pará. Nativa, Sinop, 7(1): 101-108.

Pinheiro KAO, Carvalho JOP, Quanz B, Francez LMB, Schwartz G (2007) Fitossociologia de uma área de preservação permanente no leste da Amazônia: indicação de espécies para recuperação de áreas alteradas. Floresta $37: 175-187$.
Raimundo Junior COA, Keller MM, Ramos JFF, Beldini TP, Crill PM, Camargo PB, Haren JV (2015) Chemical analysis of rainfall and throughfall in the Tapajos National Forest, Belterra, Pará, Brazil. Revista Ambiente \& Água, 10(2): 264-285.

Ripley BD (1979) Tests ofrandomness' for spatial point patterns. Journal of the Royal Statistical Society. Series B (Methodological), 41(3): 368-374.

Rowlingson B, Diggle P (2016) Splancs: spatial and space-time point pattern analysis.

Austria: R Development Core Team (R package version 2.01-15)

Seidler TG, Plotkin JB (2006) Seed Dispersal and Spatial Pattern in Tropical Trees. PLoS Biology, 4(11): 21322137.

SEMAS, Secretaria de Estado de Meio Ambiente e Sustentabilidade (2015) Instrução Normativa $N^{o} 5$.

Shanley P, Medina G (2005) Frutíferas e plantas úteis na vida amazônica. CIFOR, 300p

Silva JGM (2010) Desempenho e amostragem do trabalho de uma serraria no município de Alegre, Espírito Santo. Trabalho de Conclusão do Curso, Universidade Federal do Espírito Santo, $62 \mathrm{p}$.

Silva SMM (2009) Estrutura populacional de jatobá em florestas manejadas na Amazônia sul - ocidental. In: Congresso latino americano de ecologia, São Lourenço, Minas Gerais.

Soares CPB, Paula Neto F, Souza AL (2011) Dendrometria e Inventário Florestal. $2^{\mathrm{a}}$ ed. Editora UFV. Viçosa, $272 \mathrm{p}$.

Sontag VE (2017) Alterações na legislação brasileira de manejo florestal e seus efeitos na distribuição espacial e polinização de espécies madeireiras amazônicas. Dissertação (Mestrado em Conservação de Ecossistemas Florestais), Escola Superior de Agricultura de Luiz de Queiroz, Piracicaba.

Souza AL, Soares CPB (2013) Florestas nativas: estrutura, dinâmica e manejo. Ed. UFV.

Schwartz G, Lopes JC, Kanashiro M, Mohren GM, PeñaClaros M (2014) Disturbance level determines the regeneration of commercial tree species in the Eastern Amazon. Biotropica 46:148-156.

Vieira, DS; Oliveira, MLR; Gama, JRV; Machado, ELM; Gorgens, EB (2017) Patrón espacial y métodos de muestreo para Bertholletia excelsa (castaña de Pará) en la Mesorregión del Bajo Amazonas, estado de Pará, Brasil. Bosque, 38(1): 97-107.

Vieira DS, Oliveira MLR, Gama JRV, Oliveira BL, Rego AKC, Bezerra TG (2018) Sampling processes for Carapa guianensis Aubl. in the Amazon. Cerne, 24(3): 169-179.

Wadt LHO, Kainer KA, Gomes-Silva DAP (2005) Population structure and nut yield of a Bertholletia excelsa stand in Southwestern Amazonia. Forest Ecology and Management, 211: 371-384. 